\title{
AN OPERATIONAL PROCEDURE FOR HANKEL TYPE INTEGRALS
}

\section{NASIM}

Department of Mathematics and Statistics

The University of Calgary

Calgary, Alberta T2N IN4 Canada

(Received March 28, 1986)

ABSTRACT. In this paper, an operational procedure is established to evaluate Hankel type integrals. First, an operator $L(\theta), \theta \equiv-x \frac{d}{d x}$ is constructed, which defines the integral. Then making use of some basic properties of this operator, an elementary procedure is developed for evaluating integrals for a special class of analytic functions. A few example are given to illustrate the technique.

KBY WORDS AND PHRASBS. Differential operator of infinite order, Hankel transform, Mellin transform, Bessel functions, Hypergeometric functions.

SUBJBCT CLASSIFICATION: $44 A 15$

\section{INTRODUCTION.}

We consider the singular integral of the type

$$
\int_{0}^{\infty} f(x t) J_{\nu}(2 t) d t
$$

where $J_{\nu}$ is the usual Bessel function of the first kind of order $\nu, \nu \geq-\frac{1}{2}$, and $f(x)$ is a suitable function. This integral can be viewed as defining the Hankel transform of the function $f$. In this note, our main ain is to construct an operator $L(\theta), \theta=-x \frac{d}{d x}$, so that $L(\theta)[f(x)]$ defines the integral (1.1), [cf. 1, 19.5; 2$]$. We then establish properties of the operator $L(\theta)$, and with these help of the properties, we shall obtain an operational procedure to evaluate the integral (1.1).

2. THB OPERATOR.

It is an easy watter to see that the differential operator $\theta^{n}$, where $\theta \equiv-x \frac{d}{d x}$ and n a positive integer, is such that

$$
\theta^{n}\left[x^{\alpha}\right]=(-\alpha)^{n} x^{\alpha},
$$

for some constant $\alpha$. Then $p_{n}(\theta)$, a polynomial of $n^{\text {th }}$ degree in $\theta$, gives

$$
p_{n}(\theta)\left[x^{\alpha}\right]=p_{n}(-\alpha) x^{\alpha} \text {. }
$$

Consequently, 


$$
\begin{aligned}
p(\theta)\left[x^{\alpha}\right] & =\lim _{n \rightarrow \infty} p_{n}(\rho)\left[x^{\alpha}\right] \\
& =\lim _{n \rightarrow \infty} p_{n}(-\alpha) x^{\alpha} \\
& =p(-\alpha) x^{\alpha},
\end{aligned}
$$

where $p(s)=\lim _{n \rightarrow \infty} p_{n}(s)$, limit of a polynomial. Thus the operator $p(\theta)$, is a differential operator of infinite order and has the property that when applied to a power function, simply replaces it with a multiplier. With this understanding, we may write, symbolically,

$$
n^{-\theta}=e^{-\theta \ln }=\lim _{N \rightarrow \infty} \sum_{k=0}^{N} \frac{(-\ln n)^{k}}{k !} \theta^{k}=\lim _{N \rightarrow \infty} p_{N}(\theta) \text {, say. }
$$

Then,

$$
\begin{aligned}
n^{-\theta}\left[x^{-s}\right] & =\lim _{N \rightarrow \infty} p_{n}(\theta)\left[x^{-s}\right]=\lim _{N \rightarrow \infty} p_{n}(s) x^{-s} \\
& =n^{-s} x^{-s}
\end{aligned}
$$

for some $s=0+i r,-\infty<\tau<\infty$.

If further

$$
\begin{aligned}
p_{n}(\theta) & ={\underset{k=1}{I I}(\nu-1+2 k+\theta), \text { and } s=o+i r, \text { then }}^{P_{n}(\rho)\left[x^{-s}\right]=\prod_{k=1}^{n}(\nu-1+2 k+\theta)\left[x^{-s}\right]} \\
& =\prod_{k=1}^{n}(\nu-1+2 k+s) x^{-s}
\end{aligned}
$$

Next we write

$$
\begin{aligned}
x^{-s} & =\frac{a-\theta}{a-\theta}\left[x^{-s}\right] \\
& =\frac{1}{a-\theta}(a-\theta)\left[x^{-s}\right]=\frac{1}{a-\theta}(a-s) x^{-8}
\end{aligned}
$$

or,

$$
\frac{1}{a-\theta}\left[x^{-s}\right]=\left(\frac{1}{a-s}\right) x^{-s} \text {, }
$$

for some constants and $s$. This defines an operator of the type $\frac{1}{a-\theta}$, in the sense that when applied to a power function, simply reproduces it with a multiplier. This property parallels that of the operator which is a polynomial or limit of a polynomial in $\theta$. By a repeated application of an operator of the type (2.3), we have

$$
\prod_{k=1}^{n}\left(\frac{1}{\nu-1+2 k-8}\right)\left[x^{-s}\right]=\underset{k=1}{n}\left(\frac{1}{\nu-1+2 k-s}\right) x^{-8} \text {, }
$$

for some $s$, except where $s=\nu-1+2 k, k=1,2, \ldots, n$.

Combining the results $(2.1),(2.2)$ and $(2.4)$, we construct the operator

$$
k_{n}^{*}(1-\theta)=\frac{1}{2} n^{-\theta} \underset{k=1}{n}\left(\frac{\nu-1+2 k+\theta}{\nu-1+2 k-\theta}\right)
$$

such that

$$
k_{n}^{*}(1-\theta)\left[x^{-s}\right]=k_{n}^{*}(1-s) x^{-s}
$$

where

$$
k_{n}^{*}(1-s)=\frac{1}{2} n^{-s} \prod_{k=1}^{n}\left(\frac{\nu-s+2 k+s}{\nu-1+2 k-s}\right), s=0+i \tau,-\infty<\tau<\infty .
$$


Next, we shall establish properties of the function $k_{n}^{*}(1-8)$.

LEMMA 1. $\left|k_{n}^{*}(1-\sigma-i \tau)\right|=n^{-0} 0(1)$, as $|r| \rightarrow \infty$.

This is quite obvious.

LEMMA 2. $\lim _{n \rightarrow \infty} k_{n}^{*}(1-s)$ exists and is uniform on every compact set of the s-axis.

ProOF. From above,

$$
\begin{aligned}
& k_{n}^{*}(1-s)=\frac{1}{2} e^{-s \ln n} \underset{k=1}{n}\left(\frac{v-1+2 k+s}{v-1+2 k-s}\right) \\
& =\frac{1}{2} e^{s\left(1+\frac{1}{2}+\cdots+\frac{1}{n} \ln n\right)} \underset{k=1}{n}\left(1+\frac{2 s}{\nu-1+2 k-8}\right) e^{-s / k} .
\end{aligned}
$$

Consider the product

$$
\begin{aligned}
{\underset{k=1}{n}\left(1+\frac{2 s}{\nu-1+2 k-s}\right) e^{-s / k}}^{n} & =\prod_{k=1}^{n}\left[1+\left(1+\frac{2 s}{\nu-1+2 k-s}\right) e^{-s / k}-1\right] \\
& =\prod_{k=1}^{n}\left[1+a_{k}(s)\right] .
\end{aligned}
$$

Now,

$$
\begin{aligned}
\lim _{k \rightarrow \infty} k^{2} a_{k}(s) & =\lim _{k \rightarrow \infty} k^{2}\left[\left(1+\frac{2 s}{\nu-1+2 k-s}\right) e^{-s / k}-1\right] \\
& =\lim _{k \rightarrow \infty} k^{2}\left[\left(1+\frac{2 s}{\nu-1+2 k-s}\right)\left(1-\frac{s}{k}+\frac{1}{2 !} \frac{s^{2}}{k^{2}}-\cdots\right)-1\right] \\
& =\lim _{k \rightarrow \infty} k^{2}\left[s\left(\frac{2}{\nu-1+2 k-s}-\frac{1}{k}\right)+s^{2}\left(\frac{1}{2 k^{2}}-\frac{2}{k(\nu-1+2 k-s)}\right)+0\left(\frac{1}{k^{3}}\right)\right] \\
& =\frac{1}{2} s(1-\nu) .
\end{aligned}
$$

or, $a_{k}(s)=\frac{1}{2}(1-\nu) \circ(1)$, as $k \rightarrow \infty$ for all finite $s$, hence the infinite product convergence uniformly on every compact set of the s-axis. Also

$$
e^{s\left(1+\frac{1}{2}+\cdots+\frac{1}{n} \ln n\right)} \rightarrow e^{r s} \text {, as } n \rightarrow \infty
$$

$\boldsymbol{r}$ being the Euler's constant; hence the result.

$$
\begin{aligned}
& \text { In fact, } \quad \lim _{n \rightarrow \infty} k_{n}^{*}(1-s)=\lim _{n \rightarrow \infty} \frac{1}{2} n^{-8} \prod_{k=1}^{n}\left(\frac{\nu-1+2 k+s}{\nu-1+2 k-8}\right)
\end{aligned}
$$

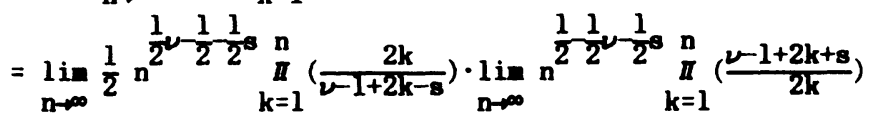

$$
\begin{aligned}
& =\frac{1}{2} \frac{\Gamma\left(\frac{1}{2}+\frac{1}{2} \nu-\frac{1}{2} s\right)}{\Gamma\left(\frac{1}{2}+\frac{1}{2}++\frac{1}{2} s\right)}=k^{*}(1-s) \text {, say, }
\end{aligned}
$$

using Buler's product for $r$-functions, $[3$, p. 11$]$.

Note that the function

$$
\begin{aligned}
k^{*}(s) & =\frac{1}{2} \frac{\Gamma\left(\frac{1}{2} \nu+\frac{1}{2} s\right)}{\Gamma\left(1+\frac{1}{2} \nu \frac{1}{2} s\right)} \\
& =M\left[J_{\nu}(2 x), s\right]
\end{aligned}
$$

the Mellin transform of $J_{\nu}(2 x), s=\sigma+i \tau,-\infty<\tau<\infty$, and $0<0<\nu+1,[4, p .326]$. Now, we define the operator 


$$
k^{*}(1-\theta)=\frac{1}{2} \frac{r\left(\frac{1}{2}+\frac{1}{2} \nu-\frac{1}{2} \theta\right)}{r\left(\frac{1}{2}+\frac{1}{2} \nu+\frac{1}{2} \theta\right)}, \theta \equiv-x \frac{d}{d x}
$$

LEMMA 3. $\mathrm{K}^{*}(1-\theta)\left[\mathrm{x}^{-s}\right]=\mathrm{k}^{*}(1-\mathrm{s}) \mathrm{x}^{-\mathrm{s}}$, where $\mathrm{k}^{*}(1-\mathrm{s})$ is defined above.

PROOF. $\quad k^{*}(1-\theta)\left[x^{-s}\right]=\lim _{n \rightarrow \infty} k_{n}^{*}(1-\theta)\left[x^{-8}\right]$, where by using Euler's product for $r$-functions, $k^{*}(1-\theta)=\lim _{n \rightarrow \infty} k_{n}^{*}(1-\rho)=\frac{1}{2} \lim _{n \rightarrow \infty} n^{-\theta} \underset{k=1}{n}\left(\frac{\nu-1+2 k+\theta}{\nu-1+2 k-\theta}\right)$, as in (2.5) above.

Now, using the results (2.6) and (2.7) we have

$$
\begin{aligned}
k^{*}(1-\theta)\left[x^{-8}\right] & =\lim _{n \rightarrow \infty} k_{n}^{*}(1-\theta)\left[x^{-s}\right] \\
& =\lim _{n \rightarrow \infty} k_{n}^{*}(1-s) x^{-s} \\
& =k^{*}(1-s) x^{-s}
\end{aligned}
$$

as desired.

3. THB INTBGRAL.

THEOREM 1. Let $f(x)$ be such that $f^{*}(s)=\mu[f(x): s] \in L(\sigma-i \infty, \sigma+i \infty)$ and $k(x)=J_{\nu}(2 x), \nu \geq-\frac{1}{2}$ and $0<0<\nu+1$. Then

$$
k^{*}(1-\theta)[f(x)]=\int_{0}^{\infty} f(x t) J_{\nu}(2 t) d t,
$$

where $k^{*}(1-\theta)$ is the operator defined by $(2.8)$ above.

PROOF. Since $f^{*}(s)$ defines the Mellin transform of $f(x)$, we may write

$$
\begin{aligned}
k^{*}(1-\theta)[f(x)]=k^{*} & (1-\theta) \frac{1}{2 \pi i} \int_{\sigma-i \infty}^{\sigma+i^{\infty}} f^{*}(s) x^{-s} d s \\
& =\lim _{n \rightarrow \infty} k_{n}^{*}(1-\theta) \frac{1}{2 \pi i} \int_{\sigma-i \infty}^{\sigma+i \infty} f^{*}(s) x^{-s} d s \\
& =\lim _{n \rightarrow \infty} \frac{1}{2 \pi i} \int_{\sigma-i \infty}^{\sigma+i \infty} f^{*}(s) k_{n}^{*}(1-\theta)\left[x^{-s}\right] d s \\
& =\lim _{n \rightarrow \infty} \frac{1}{2 \pi i} \int_{\sigma-i \infty}^{\sigma+i \infty} f^{*}(s) k_{n}^{*}(1-s) x^{-s} d s,
\end{aligned}
$$

due to Lemma 3 . To justify bringing the operator $k_{n}^{*}(1-\theta)$ inside the integral sign, we have simply to show that the resulting integral

$$
\int_{0-i \infty}^{\sigma+i \infty} f^{*}(s) k_{n}^{*}(1-s) x^{-s} d s
$$

is uniformly convergent for all finite $x$. This is so, since

$$
\begin{aligned}
\left|\int_{\sigma-i \infty}^{\sigma+i \infty} f^{*}(s) k_{n}^{*}(1-s) x^{-s} d s\right| & =\left|\int_{-\infty}^{\infty} f^{*}(\sigma+i \tau) k_{n}^{*}(1-\sigma-i \tau) x^{-\sigma-i \tau} i d r\right| \\
& =0\left(n^{-\sigma}\right) x^{-\sigma} \int_{-\infty}^{\infty}\left|f^{*}(\sigma+i \tau)\right| d \tau<\infty,
\end{aligned}
$$

for $x>0, \sigma>0$, since $f^{*}(s) \in L\left(\sigma-i^{\infty}, \sigma+i^{\infty}\right)$ and by using tahe results of Lemma 1 . In fact the integral (3.2) converges absolutely, as well. Together with the results of Lemma 2 and 3, we can then apply Lebesgue's limit theorem, to obtain 


$$
\begin{aligned}
k^{*}(1-\theta)[f(x)] & =\underset{n \rightarrow \infty}{\lim } \frac{1}{2 \pi i} \int_{\sigma-i \infty}^{\sigma+i \infty} f^{*}(s) k_{n}^{*}(1-s) x^{-s} d s \\
& =\frac{1}{2 \pi i} \int_{\sigma-i \infty}^{\sigma+i \infty} f^{*}(s) \underset{n-\infty}{[1 i m} k_{n}^{*}(1-s) x^{-s} d s \\
& =\frac{1}{2 \pi i} \int_{\sigma-i \infty}^{\sigma+i \infty} f^{*}(s) k^{*}(1-s) x^{-s} d s \\
& =\int_{0}^{\infty} f(x t) J_{\nu}(2 t) d t
\end{aligned}
$$

due to the Parseval theoren for Mellin transforms [5, chapt. II], and since $\mathbf{k}^{*}(\mathbf{s})=$ $\boldsymbol{u}\left[\mathrm{J}_{\boldsymbol{v}}(2 \mathrm{t}) ; \mathrm{s}\right]$. Hence the theorem.

Thus the equation (3.1) defines $k^{*}(1-\theta)$ as an integral operator, having the property that

$$
k^{*}(1-\theta)\left[x^{\alpha}\right]=k^{*}(1+\alpha) x^{\alpha},
$$

for some $\alpha$, due to Lema 3. In light of the operational calculus generated by the operator $k^{*}(1-\theta)$, one can now evaluate the integrals of the Hankel type, using the operational procedures. For instance if $f(x)$ is analytic and expressed in a power series

$$
f(x)=\sum_{n=0}^{\infty} C_{n} x^{n+\alpha},|x|<r,
$$

for some $r$ and $\alpha$, then

$$
\begin{aligned}
k^{*}(1-\theta)[f(x)] & =k^{*}(1-\theta) \sum_{n=0}^{\infty} C_{n} x^{x+\alpha} \\
& =\sum_{n=0}^{\infty} C_{n} k^{*}(1-\theta)\left[x^{n+\alpha}\right] \\
& =\sum_{n=0}^{\infty} C_{n} k^{*}(1+n+\alpha) x^{n+\alpha},|x|<R,
\end{aligned}
$$

for some $R$, where

$$
k^{*}(1+n+\alpha)=\frac{1}{2} \frac{\Gamma\left(\frac{1}{2}+\frac{1}{2} \nu+\frac{1}{2} n+\frac{1}{2} \alpha\right)}{\Gamma\left(\frac{1}{2}+\frac{1}{2} \nu-\frac{1}{2} n-\frac{1}{2} \alpha\right)} .
$$

The above analysis is justified provided

$$
\lim _{n \rightarrow \infty}\left|\frac{n C_{n+1}}{C_{n}}\right|=0(1)
$$

Hence we now have an operational procedure for evaluating the given integrals, in fact,

$$
\int_{0}^{\infty} f(x t) J_{\nu}(2 t) d t=\sum_{n=0}^{\infty} C_{n} k^{*}(1+n+\alpha) x^{n+\alpha},
$$

where $\quad f(x)=\sum_{n=0}^{\infty} C_{n} x^{n+\alpha}$ and $k^{*}(s)=\mu\left[J_{\nu}(t): s\right]=\frac{1}{2} \frac{\Gamma\left(\frac{1}{2} \nu+\frac{1}{2} s\right)}{\Gamma\left(1+\frac{1}{2} \nu-\frac{1}{2} s\right)}$.

4. SPBCIAL CASBS AND BXAMPLBS.

Consider the case when $\nu=\frac{1}{2}$. Then from (3.1), we have

$$
\frac{1}{\sqrt{\pi}} \int_{0}^{\infty} f(x t) \frac{\sin (2 t)}{\sqrt{t}} d t=k^{*}(1-\theta)[f(x)]
$$


where $k^{*}(1-\theta)=\frac{1}{2} \frac{\Gamma\left(\frac{3}{4}-\frac{1}{2} \theta\right)}{\Gamma\left(\frac{3}{4}+\frac{1}{2} \theta\right)}$. If for instance, we let $f(x)=x^{\mu},|\mu|<\frac{1}{2}$, then

$$
\begin{aligned}
\frac{x^{\mu}}{\sqrt{\pi}} \int_{0}^{\infty} t^{\mu-\frac{1}{2}} \sin (2 t) d t & =\frac{1}{2} \frac{\Gamma\left(\frac{3}{4}-\frac{1}{2} \theta\right)}{\Gamma\left(\frac{3}{4}+\frac{1}{2} \theta\right)}\left[x^{\mu}\right] \\
& =\frac{1}{2} \frac{\Gamma\left(\frac{3}{4}+\frac{1}{2} \mu\right)}{\Gamma\left(\frac{3}{4}-\frac{1}{2} \mu\right)} x^{\mu},
\end{aligned}
$$

or,

$$
\int_{0}^{\infty} t^{\mu-\frac{1}{2}} \sin (2 t) d t=\frac{\sqrt{\pi}}{2} \frac{\Gamma\left(\frac{3}{4}+\frac{1}{2} \mu\right)}{\Gamma\left(\frac{3}{4}-\frac{1}{2} \mu\right)} .
$$

Putting $\mu=-\frac{1}{2}$, gives us the classical result,

$$
\int_{0}^{\infty} \frac{\sin (2 t)}{t} d t=\frac{\pi}{2}
$$

Similarly, by letting $\nu=-\frac{1}{2}$ in (3.1), we have

$$
\frac{1}{\sqrt{\pi}} \int_{0}^{\infty} f(x t) \frac{\cos (2 t)}{\sqrt{t}} d t=k^{*}(1-\theta)[f(x)] \text {, }
$$

where

$$
\mathbf{k}^{*}(1-\theta)=\frac{1}{2} \frac{\Gamma\left(\frac{1}{4}-\frac{1}{2} \theta\right)}{\Gamma\left(\frac{1}{4}+\frac{1}{2} \theta\right)} .
$$

And if $f(x)=x^{\mu},|\mu|<\frac{1}{2}$, we have from above

$$
\int_{0}^{\infty} t^{\mu-\frac{1}{2}} \cos (2 t) d t=\frac{\sqrt{\pi}}{2} \frac{\Gamma\left(\frac{1}{4}+\frac{1}{2} \mu\right)}{\Gamma\left(\frac{1}{4}-\frac{1}{2} \mu\right)} .
$$

Putting $\mu=0$, give us the well-known result,

$$
\int_{0}^{\infty} \frac{\cos (2 t)}{\sqrt{t}} d t=\frac{\sqrt{\pi}}{2} \text {. }
$$

We now consider a few examples to illustrate the procedure given in formula (3.3).

1. Let $f(x)=x^{\mu} e^{-x}$. Then from (3.3), we have

$$
\int_{0}^{\infty}(x t)^{\mu} e^{-x t} J_{\nu}(2 t) d t=\frac{1}{2} \sum_{n=0}^{\infty} \frac{(-1)^{n} \Gamma\left(\frac{1}{2}+\frac{1}{2} \nu+\frac{1}{2} \mu+\frac{1}{2} n\right)}{n ! r\left(\frac{1}{2}+\frac{1}{2} \nu-\frac{1}{2} \mu-\frac{1}{2} n\right)} x^{n+\mu} .
$$

The series on the right-hand side converges absolutely for $|x|<2$, where $|\nu|<1+\mu$ and $|\nu| \geq-\frac{1}{2}$. Using the functional equation $r(z) r(1-z)=\frac{\pi}{\sin \pi z}$, and splitting the series into odd and even terms. We obtain from the right hand side of (4.3),

$$
\begin{aligned}
& \frac{1}{2 \sqrt{\pi}} \cos \frac{\pi}{2}(\mu-\nu) \sum_{n=0}^{\infty} \frac{(-1)^{m} \Gamma\left(\frac{1}{2}+\frac{1}{2} \nu+\frac{1}{2} \mu+n\right) \Gamma\left(\frac{1}{2}-\frac{1}{2} \nu+\frac{1}{2} \mu+n\right)}{n ! \Gamma\left(n+\frac{1}{2}\right)}\left(\frac{x}{2}^{2}\right)^{2 n} \\
&+\frac{1}{2 \sqrt{\pi}} \sin \frac{\pi}{2}(\mu-\nu) \sum_{n=0}^{\infty} \frac{(-1)^{n} \Gamma\left(1+\frac{1}{2} \nu+\frac{1}{2} \mu+n\right) \Gamma\left(1-\frac{1}{2} \nu+\frac{1}{2} \mu+n\right)}{n ! r\left(n+\frac{3}{2}\right)}\left(\frac{x}{2}\right)^{2 n+1}
\end{aligned}
$$

Hence, 


$$
\begin{aligned}
\int_{0}^{\infty} t^{\mu} e^{-x t} J_{\nu}(2 t) d t & =\frac{1}{2 \pi} \cos \frac{\pi}{2}(\mu-\nu) \Gamma\left(\frac{1}{2}+\frac{1}{2} \nu+\frac{1}{2} \mu\right) \Gamma\left(\frac{1}{2}-\frac{1}{2} \nu+\frac{1}{2}+\mu\right){ }_{2} F_{1}\left(\frac{1}{2}+\frac{1}{2} \nu+\frac{1}{2} \mu, \frac{1}{2}-\frac{1}{2} \nu+\frac{1}{2} \mu ; \frac{1}{2} ;-\frac{x^{2}}{4}\right) \\
& +\frac{1}{2 \pi} \sin \frac{\pi}{2}(\mu-\nu) \Gamma\left(1+\frac{1}{2} \nu+\frac{1}{2} \mu\right) \Gamma\left(1-\frac{1}{2} \nu+\frac{1}{2} \mu\right) x_{2} F_{1}\left(1+\frac{1}{2} \nu+\frac{1}{2} \mu, 1-\frac{1}{2} \nu+\frac{1}{2} \mu ; \frac{3}{2} ;-\frac{x^{2}}{4}\right)
\end{aligned}
$$

due to the result (3.3). Some special cases of this result such as when $\mu=\nu$ and $\mu=0$ can easily be derived. The range of the result (4.4) can be extended to $x>0$, by analytic continuation.

2. Let $f(x)=x^{-\lambda_{J}}(n)$. Then from (3.3), we have

$$
\int_{0}^{\infty}(x t)^{-\lambda} J_{\nu}(x t) J_{\nu}(2 t) d t=\sum_{n=0}^{\infty} \frac{(-1)^{n} \Gamma\left(\frac{1}{2}+\frac{1}{2} \nu+\frac{1}{2} \mu-\frac{1}{2} \lambda+n\right) x^{2 n+\mu-\lambda}}{n ! 2^{1+2 n+\mu} \Gamma(\mu+n+1) \Gamma\left(\frac{1}{2}+\frac{1}{2} \nu-\frac{1}{2} \mu+\frac{1}{2} \lambda-n\right)} .
$$

Now,

$$
\begin{gathered}
\Gamma\left(\frac{1}{2}+\frac{1}{2} \nu-\frac{1}{2} \mu+\frac{1}{2} \lambda-n\right) \Gamma\left(\frac{1}{2}-\frac{1}{2} \nu+\frac{1}{2} \mu-\frac{1}{2} \lambda+n\right)=\frac{\pi}{(-1)^{m} \sin \frac{\pi}{2}(1-\nu+\mu-\lambda)} \\
=(-1) \Gamma\left(\frac{1}{2}-\frac{1}{2} \nu+\frac{1}{2} \mu-\frac{1}{2} \lambda\right) \Gamma\left(\frac{1}{2}+\frac{1}{2} \nu-\frac{1}{2} \mu+\frac{1}{2} \lambda\right)
\end{gathered}
$$

Therefore the right hand side of (4.5), then gives

$$
\begin{aligned}
& =\frac{2^{-\mu-1} x^{\mu-\lambda}}{\Gamma\left(\frac{1}{2} \frac{1}{2} \nu+\frac{1}{2} \mu-\frac{1}{2} \lambda\right) \Gamma\left(\frac{1}{2}+\frac{1}{2} \nu-\frac{1}{2} \mu+\frac{1}{2} \lambda\right)} \cdot \sum_{n=0}^{\infty} \frac{\Gamma\left(\frac{1}{2}-\frac{1}{2} \nu+\frac{1}{2} \mu-\frac{1}{2} \lambda+n\right) \Gamma\left(\frac{1}{2}+\frac{1}{2} \nu+\frac{1}{2} \mu-\frac{1}{2} \lambda+n\right)}{n ! \Gamma(1+\mu+n)}\left(\frac{x^{2}}{4}\right)^{n} \\
& =\frac{2^{-\mu-1} \Gamma\left(\frac{1}{2}+\frac{1}{2} \nu+\frac{1}{2} \mu-\frac{1}{2} \lambda\right) x^{\mu-\lambda}}{\Gamma\left(\frac{1}{2}+\frac{1}{2}-\frac{1}{2} \mu+\frac{1}{2} \lambda\right) \Gamma(1+\mu)} 2_{1}^{F_{1}}\left(\frac{1}{2}-\frac{1}{2} \mu+\frac{1}{2} \mu-\frac{1}{2} \lambda, \frac{1}{2}+\frac{1}{2} \nu+\frac{1}{2} \mu-\frac{1}{2} \lambda ; 1+\mu ; \frac{x^{2}}{4}\right),
\end{aligned}
$$

where

$$
|x|\langle 2, \operatorname{Re}(\mu+\nu)+1>\operatorname{Re} \lambda>-1,[6, p .48] .
$$

As a special case if $\lambda=\nu-\mu-1$, then

$$
\begin{aligned}
\int_{0}^{\infty}(x t)^{-\nu+\mu+1} J_{\mu}(x t) J_{\nu}(2 t) d t & =\frac{2^{-\mu-1} x^{2 \mu-\nu+1}}{\Gamma(\nu-\mu)} 2^{F_{1}} 1^{\left(1-\nu+\mu, 1+\mu ; 1+\mu ; \frac{x^{2}}{4}\right)} \\
& =\frac{2^{-\mu-1} x^{2 \mu-\nu+1}}{\Gamma(\nu-\mu)}\left(1-\frac{x^{2}}{4}\right),|x|<2 .
\end{aligned}
$$

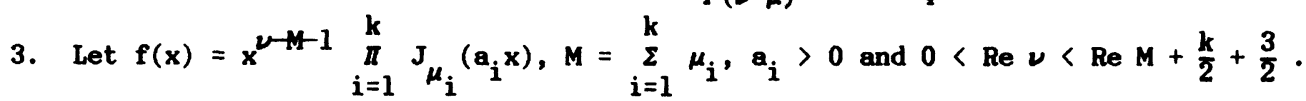
or,

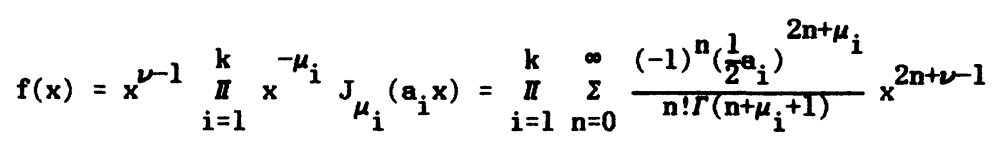

Then

$$
\begin{aligned}
& \int_{0}^{\infty}(x t)^{\nu-M-1} \prod_{i=1}^{k} J_{\mu_{i}}\left(a_{i} x t\right) J_{\nu}(2 t) d t=k^{*}(1-0)\left[\prod_{i=1}^{k} \sum_{n=0}^{\infty} \frac{(-1)^{n}\left(\frac{n_{i}}{2}\right)^{2 n+\mu_{i}}}{n ! \Gamma\left(n+\mu_{i}+1\right)} \cdot x^{2 n+\nu-1}\right] \\
& =\prod_{i=1}^{k} \sum_{n=0}^{\infty} \frac{(-1)^{n^{n}}\left(\frac{a_{i}}{2}\right)^{2 n+\mu_{i}}}{n ! \Gamma\left(n+\mu_{i}+1\right)} k^{*}(2 n+\nu) x^{2 n+\nu-1}
\end{aligned}
$$




$$
\begin{aligned}
& =\frac{1}{2} \prod_{i=1}^{k} \sum_{n=0}^{\infty} \frac{(-1)^{n^{n}}\left(\frac{a_{i}}{2}\right)^{2 n+\mu_{i}} \Gamma(\nu+n)}{n ! \Gamma\left(n+\mu_{i}+1\right) \Gamma(1-n)} x^{2 n+\nu-1}
\end{aligned}
$$

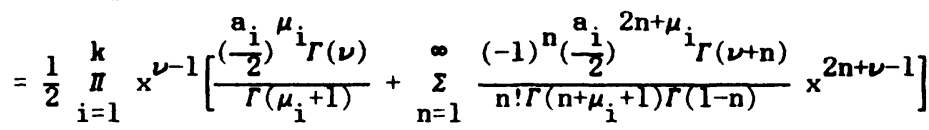

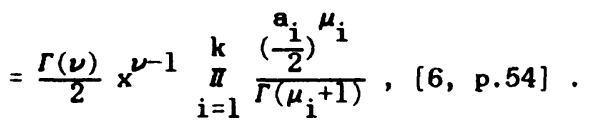

4. Finally we will derive a general result formally.

Let

$$
f(x)=x^{2 \lambda} \underset{p, q}{m, n}\left[\left.c x^{2}\right|_{b_{1}, \ldots, b_{q}} ^{a_{1}, \ldots, a_{p}}\right], p+q<2(m+n)
$$

Then

$$
\begin{aligned}
& \int_{0}^{\infty} f(x t) J_{\nu}(2 t) d t=k^{*}(1-\theta)[f(x)]
\end{aligned}
$$

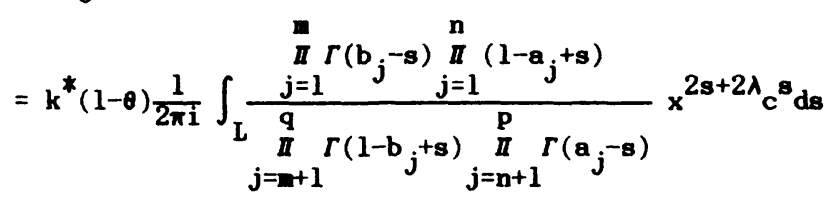

$$
\begin{aligned}
& \underset{j=1}{\operatorname{m}} \Gamma\left(\mathbf{b}_{\mathrm{j}}-\mathbf{s}\right) \underset{j=1}{\boldsymbol{n}} \Gamma\left(1-\mathbf{a}_{\mathrm{j}}+\mathbf{s}\right)
\end{aligned}
$$

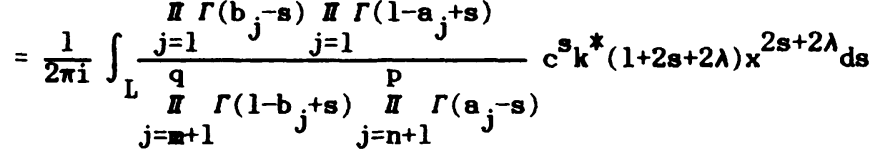

$$
\begin{aligned}
& \prod_{j=1}^{m} \Gamma\left(b_{j}-s\right) \underset{j=1}{n} \Gamma\left(1-a_{j}+s\right) \Gamma\left(\frac{1}{2}+\frac{1}{2} \nu+s+\lambda\right)
\end{aligned}
$$

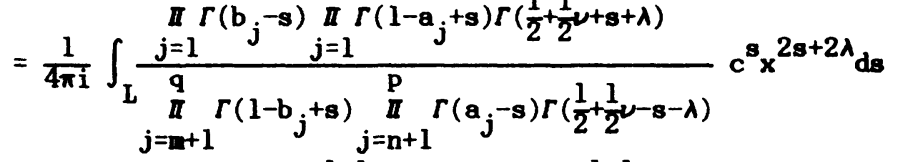

$$
\begin{aligned}
& =\frac{1}{2} x^{2 \lambda} G_{p+2, q}^{m, n+1}\left[\left.c x^{2}\right|_{b_{1}, \ldots, b_{q}} ^{\frac{1}{2}-\frac{1}{2} \nu-\lambda, a_{1}, \ldots, a_{p}, \frac{1}{2}+\frac{1}{2} \nu-\lambda}\right] \text {, }
\end{aligned}
$$

with the usual conditions on paraneters, $[6$, p.91 $]$.

\section{REFERBANCBS}

1. WIDDER, D.V. An Introduction to Transforn Theory, Academic Press, New York, (1971).

2. NASIM, C. An inversion formula for Hankel transforms, Pacific J. Vol. 57 (1), 1975 Pp. 255-258.

3. RAINVILLB, B.D. Special Functions, Macmillan, New York, (1967).

4. ERDELYI, A. et al. Tables of integral transforns Vol. I, McGraw-Hill, New York, (1954).

5. TITCHMARH, B.C. An Introduction to the Theory of Integral Transfores, Second Bdition, Oxford University Press, (1948). 


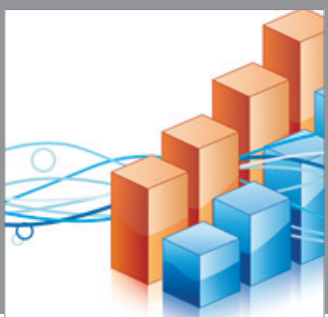

Advances in

Operations Research

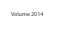

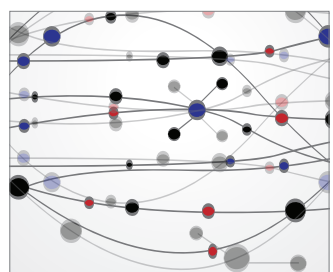

\section{The Scientific} World Journal
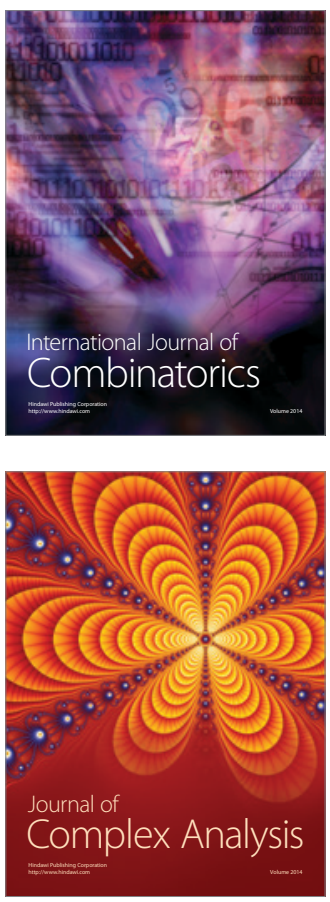

International Journal of

Mathematics and

Mathematical

Sciences
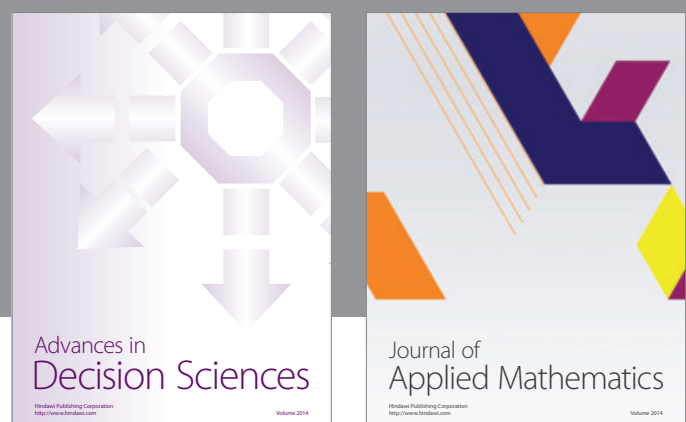

Journal of

Applied Mathematics
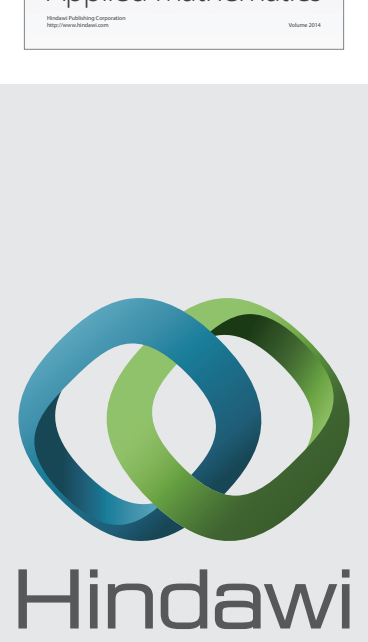

Submit your manuscripts at http://www.hindawi.com
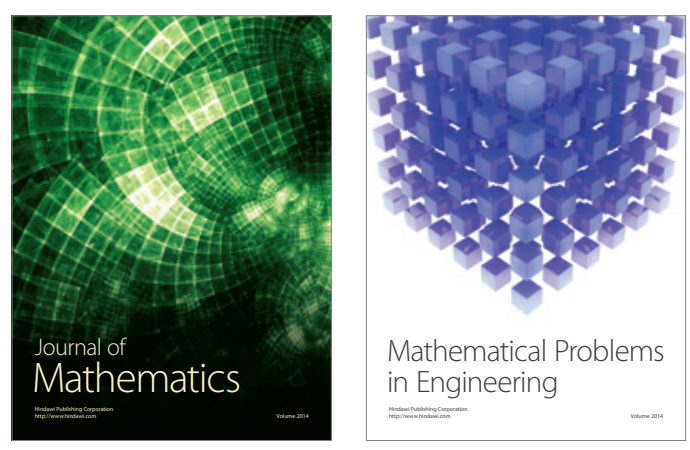

Mathematical Problems in Engineering
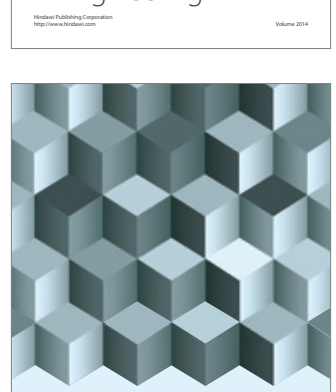

Journal of

Function Spaces
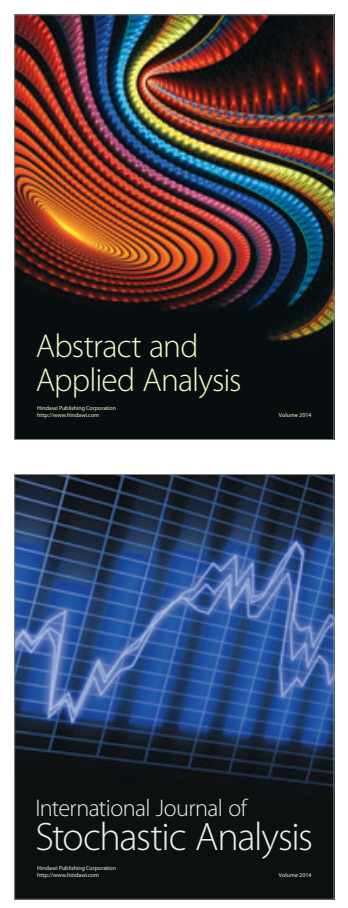

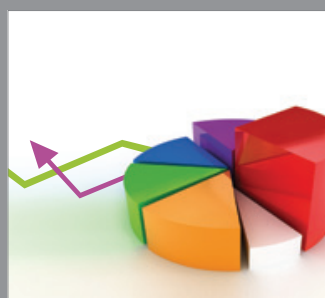

ournal of

Probability and Statistics

Promensencen
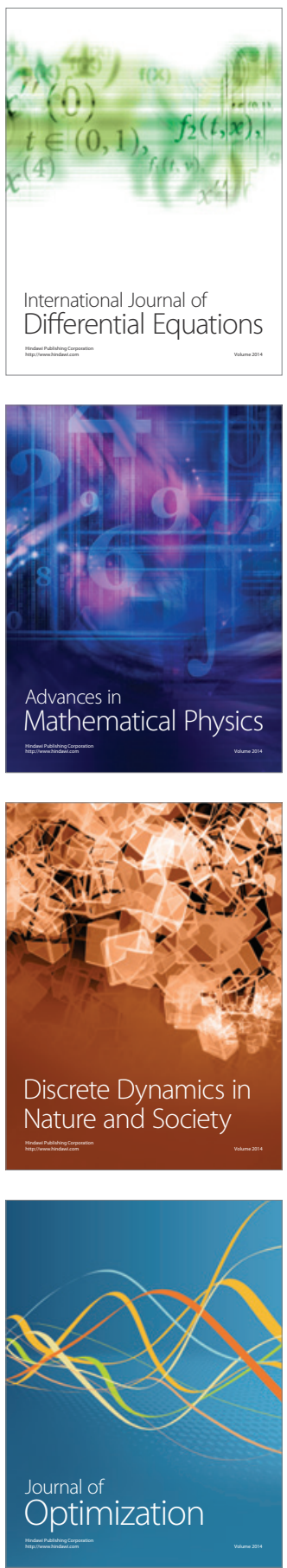